\title{
What Factors Increase the Risk of Complications in SARS-CoV-2-Infected Patients? A Cohort Study in a Nationwide Israeli Health Organization
}

Chen Yanover ${ }^{1 *}$, PhD; Barak Mizrahi ${ }^{1 *}$, MSc; Nir Kalkstein ${ }^{1}$, BSc; Karni Marcus ${ }^{1}$, MD, MBA; Pinchas Akiva ${ }^{1}, \mathrm{PhD}$; Yael Barer ${ }^{2}$, MSc; Varda Shalev², MD; Gabriel Chodick ${ }^{2,3}, \mathrm{PhD}$

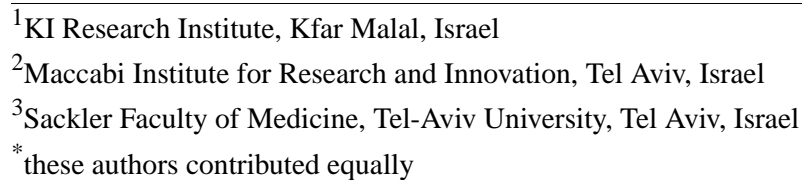

\section{Corresponding Author:}

Chen Yanover, PhD

KI Research Institute

11 Hazayit St

Kfar Malal, 4592000

Israel

Phone: 972545493154

Email: chen@kinstitute.org.il

\begin{abstract}
Background: Reliably identifying patients at increased risk for coronavirus disease (COVID-19) complications could guide clinical decisions, public health policies, and preparedness efforts. Multiple studies have attempted to characterize at-risk patients, using various data sources and methodologies. Most of these studies, however, explored condition-specific patient cohorts (eg, hospitalized patients) or had limited access to patients' medical history, thus, investigating related questions and, potentially, obtaining biased results.

Objective: This study aimed to identify factors associated with COVID-19 complications from the complete medical records of a nationally representative cohort of patients, with severe acute respiratory syndrome coronavirus 2 (SARS-CoV-2) infection.

Methods: We studied a cohort of all SARS-CoV-2-positive individuals, confirmed by polymerase chain reaction testing of either nasopharyngeal or saliva samples, in a nationwide health organization (covering 2.3 million individuals) and identified those who suffered from serious complications (ie, experienced moderate or severe symptoms of COVID-19, admitted to the intensive care unit, or died). We then compared the prevalence of pre-existing conditions, extracted from electronic health records, between complicated and noncomplicated COVID-19 patient cohorts to identify the conditions that significantly increase the risk of disease complications, in various age and sex strata.

Results: Of the 4353 SARS-CoV-2-positive individuals, 173 (4\%) patients suffered from COVID-19 complications (all age $\geq 18$ years). Our analysis suggests that cardiovascular and kidney diseases, obesity, and hypertension are significant risk factors for COVID-19 complications. It also indicates that depression (eg, males $\geq 65$ years: odds ratio [OR] 2.94, 95\% CI 1.55-5.58; $P=.01$ ) as well as cognitive and neurological disorders (eg, individuals $\geq 65$ years old: OR $2.65,95 \%$ CI $1.69-4.17 ; P<.001$ ) are significant risk factors. Smoking and presence of respiratory diseases do not significantly increase the risk of complications.

Conclusions: Our analysis agrees with previous studies on multiple risk factors, including hypertension and obesity. It also finds depression as well as cognitive and neurological disorders, but not smoking and respiratory diseases, to be significantly associated with COVID-19 complications. Adjusting existing risk definitions following these observations may improve their accuracy and impact the global pandemic containment and recovery efforts.
\end{abstract}

(JMIR Public Health Surveill 2020;6(3):e20872) doi: 10.2196/20872

\section{KEYWORDS}

SARS-CoV-2; COVID-19; risk factors; complications 


\section{Introduction}

As of April 30, 2020, more than 3 million people worldwide contracted severe acute respiratory syndrome coronavirus 2 (SARS-CoV-2) and close to 250,000 people died of coronavirus disease (COVID-19) complications. In Israel, by that date, 16,004 individuals had been infected by the virus and 223 died from the disease. This pandemic poses grave challenges to patients, health care providers, and policy makers. Many of these challenges may be better addressed with timely stratification of patients to risk groups, based on their past and current medical characteristics. For example, reliably identifying patients at increased (or decreased) risk could guide clinical decisions (eg, hospitalization vs home care), public health policies (eg, risk-based quarantine), and preparedness efforts (eg, expected medical equipment required).

Various algorithms for identifying patients at risk for COVID-19 (severe) complications have been proposed. The Centers for Disease Control and Prevention (CDC) identified individuals 65 years and older, living in a nursing home or long-term care facility, or suffering from underlying medical conditions, particularly if not well controlled, as being at high risk for severe illness from COVID-19 [1]. Similarly, the European Centre for Disease Prevention and Control (ECDC) lists the age category $>70$ years and some underlying conditions as risk factors for critical illness [2]. The United Kingdom National Health Service (NHS) included solid organ transplant recipients, patients with specific cancers or severe respiratory conditions, pregnant women with significant heart disease, and those with increased risk of infection (eg, due to immunosuppressive therapies) in the highest clinical COVID-19 risk group [3]. In April 2020, approximately 1.3 million people in this group were asked to "shield" by staying at home for a period of at least 12 weeks. In addition, patients $>70$ years and those suffering from some underlying health conditions (eg, chronic respiratory diseases, $\mathrm{BMI} \geq 40$, and pregnant women) were considered in a wider vulnerable group (also referred to as the "flu group"). Finally, a more quantitative risk model (derived from Barda et al [4]) was adopted by the Israeli Ministry of Health $(\mathrm{MoH})$, assigning a point for each underlying condition from a predefined list, then considering age group and point count to identify high-risk patients.

Initially, these algorithms were derived from a quickly growing number of epidemiological characterization studies (eg, [5,6]), which report the prevalence of various conditions in a population of interest, typically severe, hospitalized COVID-19 patients. These studies provide timely and important information; however, identifying risk factors calls for a comparative analysis, contrasting the prevalence of conditions in case and control populations. To date, only a handful of studies implemented such an approach, using, for example, the general population [7] or a confirmed (and symptomatic) COVID-19 patient cohort [8]. Similar to these efforts, we analyze here the medical records of all SARS-CoV-2-positive patients in a nationwide health organization (covering 2.3 million individuals). We compare the prevalence of existing conditions in complicated and noncomplicated cohorts and identify those conditions associated with COVID-19 complications in various age and sex strata. Our analysis highlights stratum-specific risk factors and may allow better identification of patients at risk in different subpopulations.

\section{Methods}

\section{Data Source}

Maccabi Health Services (MHS) is a nationwide health plan (payer-provider), representing a quarter of the Israeli population. The MHS database contains longitudinal data on a stable population of over 2.3 million people since 1993 (with an annual attrition rate lower than $1 \%$ ). Data are automatically collected and include comprehensive laboratory data from a single central lab, full pharmacy prescription and purchase data, and extensive demographic information on each patient.

Data are available upon reasonable request. According to Israeli regulations, no patient-level secondary use medical data can be publicly shared.

\section{Study Design and Setting}

SARS-CoV-2 polymerase chain reaction testing in Israel uses both nasopharyngeal and saliva samples. Individuals with a positive test result (as of April 22, 2020) were included in the SARS-CoV-2-positive cohort. Positive patients whose disease status, as updated by Israeli hospitals, deteriorated to moderate or severe (at any point in time), admitted to the intensive care unit, or died constitute the complicated COVID-19 cohort. Initially, the definition of disease status varied, to some extent, between hospitals but was largely based on the severity of lower respiratory tract symptoms, including pneumonia, respiratory distress, and artificial respiration, as well as shock and system failure. The remaining SARS-CoV-2-positive patients (including asymptomatic, mild COVID-19 patients, or those with unknown status) constitute the noncomplicated COVID-19 cohort. The follow-up period ended on April 30, 2020 (or upon patient's death).

Patients nor the public were involved in the design, or conduct, or reporting, or dissemination plans of our research.

\section{Patient Characteristics}

Apart from age and sex, we considered a set of existing conditions, comprising those included in the CDC, NHS, and Israeli $\mathrm{MoH}$ at-risk definitions, as well as a set of conditions showing significant association with flu and flu-like complications.

To identify each individual's existing conditions, we used, when available, registries created and maintained by MHS. These registries are based on validated inclusion and exclusion criteria (considering coded diagnoses, treatments, labs, and imaging, as applicable). The registries are continuously and retrospectively (since 1998) updated based on each patient's central medical record. Patients may be excluded from a registry when deemed misclassified by their primary physician. Linkage across registries and with other sources of information is performed via a unique national identification number. MHS registries used are: cardiovascular diseases (specifically, ischemic heart disease, congestive heart failure, peripheral vascular disease, cerebrovascular disease, and other 
cardiovascular diseases) [9], diabetes [10,11], hypertension [12], osteoporosis [13], chronic kidney disease [14], cognitive disorders, mental illness [15], cancer, immunosuppression (including advanced kidney disease, immunosuppressive treatment, asplenia, and organ transplant), weight disorders (obesity, overweight, and underweight), smoking, hospitalization (in the last 3 years), nursing home, and home care (home visits, home respiratory care, respiratory and feeding equipment). For other conditions, we relied on previously grouped lists of diagnosis codes (Read codes or International Classification of Diseases codes, 9th revision) [16-18]: deficiency anemia, fluid and electrolyte disorders, respiratory diseases (specifically, chronic obstructive pulmonary disease, chronic pulmonary disease, pleural effusion, aspiration pneumonia, and bronchiectasis), neurological disorders, end stage renal disease, rheumatoid arthritis, paralysis, hip fracture, lymphoma, and alcohol consumption.

\section{Statistical Analysis}

We extracted the prevalence of the studied conditions (excluding ones with less than 20 occurrences) in the noncomplicated and complicated COVID-19 cohorts and measured the association between each condition and disease complication by computing the corresponding odds ratio (OR) and its estimated statistical significance (using Fisher exact test). We conducted the analysis separately in three age groups (18-50 years, 50-65 years, and $\geq 65$ years), as well as four (age, sex) strata (male or female; younger or older than 65 years). Using different age groups (as sensitivity analysis) obtained similar results. Finally, to account for multiple testing, we controlled for the false discovery rate using Benjamini and Hochberg's method [19]. All analyses were performed using version 4.0.0 of the $\mathrm{R}$ programming language (R Project for Statistical Computing; R Foundation). We used the STROBE (Strengthening The Reporting of OBservational Studies in Epidemiology) cohort checklist when writing our report [20].

\section{Ethical Approval}

The study was approved by the institutional review board of MHS (0024-20-MHS).

\section{Results}

The MHS SARS-CoV-2-positive cohort included 4353 individuals, of whom 173 deteriorated to moderate $(n=87,50 \%)$ or severe condition $(n=45,26 \%)$, were admitted to the intensive care unit ( $\mathrm{n}=66,38 \%$, partly overlapping with other conditions), or died $(n=21,12 \%)$. This group of patients make up the complicated COVID-19 cohort. Overall, patients in the complicated COVID-19 cohort were older, suffered from more comorbidities, and were predominantly male (Table 1). Moreover, the prevalence of COVID-19 complications increased with age and more steeply for men than for women (Table 2). The risk of COVID-19 complications in men $<70$ years was significantly higher than in women (eg, $P=.01$ for patients $60-70$ years old; see Table 2). 
Table 1. Characteristics of the severe acute respiratory syndrome coronavirus 2 (SARS-CoV-2)-positive, complicated, and noncomplicated coronavirus disease (COVID-19) patient cohorts.

\begin{tabular}{|c|c|c|c|}
\hline Characteristic & $\begin{array}{l}\text { SARS-CoV-2 positive } \\
(\mathrm{n}=4353)\end{array}$ & $\begin{array}{l}\text { Complicated COVID- } \\
19(\mathrm{n}=173)\end{array}$ & $\begin{array}{l}\text { Noncomplicated } \\
\text { COVID-19 }(\mathrm{n}=4180)\end{array}$ \\
\hline \multicolumn{4}{|l|}{ Demographic information } \\
\hline Age (years), median (IQR) & $35(22-54)$ & $70(58-80)$ & $34(22-52)$ \\
\hline$<18, \mathrm{n}(\%)$ & $647(15)$ & $0(0)$ & $647(15.6)$ \\
\hline $18-50, \mathrm{n}(\%)$ & $2354(54.5)$ & $21(12.1)$ & $2333(56.3)$ \\
\hline $50-60, \mathrm{n}(\%)$ & $609(14.1)$ & $29(16.8)$ & $580(14)$ \\
\hline $60-70, \mathrm{n}(\%)$ & $376(8.7)$ & $35(20.2)$ & $341(8.2)$ \\
\hline $70-80, \mathrm{n}(\%)$ & $232(5.4)$ & $42(24.3)$ & $190(4.6)$ \\
\hline$\leq 80, \mathrm{n}(\%)$ & $135(3.1)$ & $46(26.6)$ & $89(2.1)$ \\
\hline Female, n (\%) & $1939(44.5)$ & $50(28.9)$ & $1889(45.2)$ \\
\hline Follow-up days, median (IQR) & $30(24-36)$ & $28(21-33)$ & $30(24-36)$ \\
\hline \multicolumn{4}{|l|}{ Comorbidities, n (\%) } \\
\hline Chronic respiratory diseases & $481(11)$ & $39(22.5)$ & $442(10.6)$ \\
\hline Chronic obstructive pulmonary disease & $310(7.1)$ & $24(13.9)$ & $286(6.8)$ \\
\hline Other chronic pulmonary disease & $153(3.5)$ & $10(5.8)$ & $143(3.4)$ \\
\hline Pleural effusion & $41(0.9)$ & $4(2.3)$ & $37(0.9)$ \\
\hline Cardiovascular diseases & $310(7.1)$ & $57(32.9)$ & $253(6.1)$ \\
\hline Ischemic heart disease & $132(3)$ & $27(15.6)$ & $105(2.5)$ \\
\hline Congestive heart failure & $30(0.7)$ & $11(6.4)$ & $19(0.5)$ \\
\hline Cerebrovascular disease & $57(1.3)$ & $15(8.7)$ & $42(1)$ \\
\hline Peripheral vascular disease & $23(0.5)$ & $7(4)$ & $16(0.4)$ \\
\hline Other cardiovascular diseases & $199(4.6)$ & $41(23.7)$ & $158(3.8)$ \\
\hline Hypertension & $627(14.4)$ & $102(59)$ & $525(12.6)$ \\
\hline Immunosuppression & $164(3.8)$ & $31(17.9)$ & $133(3.2)$ \\
\hline Cancer & $205(4.7)$ & $33(19.1)$ & $172(4.1)$ \\
\hline Deficiency anemia & $423(9.7)$ & $18(10.4)$ & $405(9.7)$ \\
\hline \multicolumn{4}{|l|}{ Liver and kidney diseases } \\
\hline Liver disease & $404(9.3)$ & $28(16.2)$ & $376(9)$ \\
\hline Chronic kidney disease & $384(8.8)$ & $86(49.7)$ & $298(7.1)$ \\
\hline End stage renal disease & $85(2)$ & $26(15)$ & $59(1.4)$ \\
\hline Fluid and electrolyte disorders & $394(9.1)$ & $37(21.4)$ & $357(8.5)$ \\
\hline \multicolumn{4}{|l|}{ Metabolic diseases } \\
\hline Diabetes & $362(8.3)$ & $58(33.5)$ & $304(7.3)$ \\
\hline Obesity (BMI $\geq 30)$ & $874(20.1)$ & $73(42.2)$ & $801(19.2)$ \\
\hline \multicolumn{4}{|l|}{ Neurological and cognitive disorders } \\
\hline Neurological disorders & $294(6.8)$ & $57(32.9)$ & $237(5.7)$ \\
\hline Paralysis & $53(1.2)$ & $12(6.9)$ & $41(1)$ \\
\hline Depression & $578(13.3)$ & $53(30.6)$ & $525(12.6)$ \\
\hline Cognitive impairment & $87(2)$ & $28(16.2)$ & $59(1.4)$ \\
\hline \multicolumn{4}{|l|}{ Other } \\
\hline Hospitalization & $931(21.4)$ & $92(53.2)$ & $839(20.1)$ \\
\hline
\end{tabular}




\begin{tabular}{|c|c|c|c|}
\hline Characteristic & $\begin{array}{l}\text { SARS-CoV-2 positive } \\
(\mathrm{n}=4353)\end{array}$ & $\begin{array}{l}\text { Complicated COVID- } \\
19(\mathrm{n}=173)\end{array}$ & $\begin{array}{l}\text { Noncomplicated } \\
\text { COVID-19 }(n=4180)\end{array}$ \\
\hline Smoking & $643(14.8)$ & $41(23.7)$ & $602(14.4)$ \\
\hline Current smoker & $514(11.8)$ & $30(17.3)$ & 484 (11.6) \\
\hline Past smoker & $129(3)$ & $11(6.4)$ & $118(2.8)$ \\
\hline Nursing home & $67(1.5)$ & $23(13.3)$ & $44(1.1)$ \\
\hline Home care & $44(1)$ & $17(9.8)$ & $27(0.6)$ \\
\hline
\end{tabular}

Table 2. Association of male sex and coronavirus disease (COVID-19) complications across age groups.

\begin{tabular}{|c|c|c|c|c|c|c|}
\hline \multirow[t]{3}{*}{ Age group } & \multicolumn{4}{|c|}{ Patient counts, n (\%) } & \multirow[t]{3}{*}{$\mathrm{OR}^{\mathrm{a}}(95 \% \mathrm{CI})$} & \multirow[t]{3}{*}{$P$ value $^{\mathrm{b}}$} \\
\hline & \multicolumn{2}{|l|}{ Male } & \multicolumn{2}{|l|}{ Female } & & \\
\hline & Complicated & Noncomplicated & Complicated & Noncomplicated & & \\
\hline $18-50$ years & $18(1)$ & $1300(99)$ & $3(0.3)$ & $1033(99.7)$ & $4.77(1.39-25.32)$ & .01 \\
\hline $50-60$ years & $25(7)$ & $314(93)$ & $4(1)$ & $266(99)$ & $5.28(1.79-21.15)$ & .003 \\
\hline $60-70$ years & $29(13)$ & $202(87)$ & $6(4)$ & $139(96)$ & $3.32(1.31-10.03)$ & .01 \\
\hline $70-80$ years & $27(20)$ & $108(80)$ & $15(15)$ & $82(85)$ & $1.36(0.65-2.95)$ & .47 \\
\hline$\geq 80$ years & $24(43)$ & $32(57)$ & $22(28)$ & $57(72)$ & $1.93(0.89-4.26)$ & .15 \\
\hline
\end{tabular}

${ }^{\mathrm{a} O R}$ : odds ratio. ORs greater than 1 suggest an increased risk for COVID-19 complications in males.

${ }^{\mathrm{b}} P$ values adjusted for multiple testing using Benjamini and Hochberg's method [19].

Comparing the prevalence of existing conditions in the three age groups between the complicated and noncomplicated COVID-19 cohorts revealed multiple risk factors, including obesity for patients 18-50 years old (OR 11.09, 95\% CI 4.15-32.67; $P<.001)$, chronic kidney disease for patients 50-65 years (OR 4.06, 95\% CI 1.89-8.38; $P=.005)$, and neurological disorders (OR $2.65,95 \%$ CI $1.69-4.17 ; P<.001$ ) for patients $\geq 65$ years (for a complete list, see Table 3 and Multimedia Appendix $1)$.

Stratifying by age (below and above 65 years) and sex (Table 4 and Multimedia Appendix 1), we observed that kidney diseases are a risk factor in all strata (eg, OR 3.45, 95\% CI $1.57-8.06 ; P=.02$ in women $\geq 65$ years). Additional risk factors included hypertension in males under 65 years (OR 4.56, $95 \%$ CI $2.35-8.55 ; P<.001)$; neurological disorders in females $\geq 65$ years (OR 3.55, 95\% CI 1.68-7.74; $P=.008$ ); cognitive impairment (OR 4.18, 95\% CI 1.81-9.72; $P=.009$ ) and depression (OR 2.94, 95\% CI 1.55-5.58; $P=.01$ ) in males $\geq 65$ years. Respiratory diseases and smoking, while typically more prevalent in complicated COVID-19 patients, were not identified as significant risk factors (eg, chronic obstructive pulmonary disease in patients $\geq 65$ years: OR $1.36,95 \%$ CI $0.75-2.4 ; P=.63$ ) (see Multimedia Appendix 1). 
Table 3. Most statistically significant conditions associated with increased risk of coronavirus disease (COVID-19) complications in age-stratified patient groups.

\begin{tabular}{|c|c|c|c|c|c|c|c|}
\hline \multirow[t]{3}{*}{ Condition } & \multirow[t]{3}{*}{ Age group } & \multicolumn{4}{|l|}{ Patient counts, $\mathrm{n}$} & \multirow[t]{3}{*}{$\mathrm{OR}^{\mathrm{a}}(95 \% \mathrm{CI})$} & \multirow[t]{3}{*}{$P$ value $^{\mathrm{b}}$} \\
\hline & & \multicolumn{2}{|l|}{ With condition } & \multicolumn{2}{|c|}{ Without condition } & & \\
\hline & & Complicated & Noncomplicated & Complicated & Noncomplicated & & \\
\hline Obesity & $\begin{array}{l}18-50 \\
\text { years }\end{array}$ & 14 & 356 & 7 & 1977 & $11.09(4.15-32.67)$ & $<.001$ \\
\hline Depression & $\begin{array}{l}18-50 \\
\text { years }\end{array}$ & 7 & 229 & 14 & 2104 & $4.59(1.55-12.3)$ & .03 \\
\hline Hypertension & $\begin{array}{l}18-50 \\
\text { years }\end{array}$ & 4 & 72 & 17 & 2261 & $7.37(1.76-23.41)$ & .04 \\
\hline Liver disease & $\begin{array}{l}18-50 \\
\text { years }\end{array}$ & 5 & 125 & 16 & 2208 & $5.51(1.55-16.07)$ & .04 \\
\hline $\begin{array}{l}\text { Chronic kidney } \\
\text { disease }\end{array}$ & $\begin{array}{l}50-65 \\
\text { years }\end{array}$ & 14 & 87 & 27 & 683 & $4.06(1.89-8.38)$ & .005 \\
\hline $\begin{array}{l}\text { End stage renal } \\
\text { disease }\end{array}$ & $\begin{array}{l}50-65 \\
\text { years }\end{array}$ & 5 & 8 & 36 & 762 & $13.11(3.21-48.19)$ & .006 \\
\hline $\begin{array}{l}\text { Neurological disor- } \\
\text { ders }\end{array}$ & $\geq 65$ years & 54 & 113 & 57 & 317 & $2.65(1.69-4.17)$ & $<.001$ \\
\hline $\begin{array}{l}\text { Chronic kidney } \\
\text { disease }\end{array}$ & $\geq 65$ years & 70 & 174 & 41 & 256 & $2.51(1.6-3.97)$ & .001 \\
\hline $\begin{array}{l}\text { Other cardiovascu- } \\
\text { lar diseases }\end{array}$ & $\geq 65$ years & 36 & 70 & 75 & 360 & $2.46(1.49-4.05)$ & .006 \\
\hline $\begin{array}{l}\text { Cognitive impair- } \\
\text { ment }\end{array}$ & $\geq 65$ years & 28 & 52 & 83 & 378 & $2.45(1.4-4.22)$ & .02 \\
\hline Home care & $\geq 65$ years & 16 & 22 & 95 & 408 & $3.12(1.47-6.48)$ & .02 \\
\hline Hypertension & $\geq 65$ years & 82 & 249 & 29 & 181 & $2.05(1.27-3.4)$ & .03 \\
\hline $\begin{array}{l}\text { Cardiovascular } \\
\text { diseases }\end{array}$ & $\geq 65$ years & 50 & 129 & 61 & 301 & $1.91(1.22-2.99)$ & .03 \\
\hline Nursing home & $\geq 65$ years & 20 & 35 & 91 & 395 & $2.48(1.29-4.65)$ & .04 \\
\hline
\end{tabular}

${ }^{a}$ OR: odds ratio. ORs greater than 1 suggest an increased risk for COVID-19 complications in patients with the noted condition.

${ }^{\mathrm{b}}$ In each stratum, rows are sorted ascendingly by $P$ value. 
Table 4. Most statistically significant conditions associated with increased risk of COVID-19 complications in age- and sex-stratified patient groups.

\begin{tabular}{|c|c|c|c|c|c|c|c|}
\hline \multirow[t]{3}{*}{ Condition } & \multirow{3}{*}{$\begin{array}{l}\text { Age, sex } \\
\text { group }\end{array}$} & \multicolumn{4}{|l|}{ Patient counts } & \multirow[t]{3}{*}{$\mathrm{OR}^{\mathrm{a}}$} & \multirow[t]{3}{*}{$P$ value $^{\mathrm{b}}$} \\
\hline & & \multicolumn{2}{|l|}{ With condition } & \multicolumn{2}{|c|}{ Without condition } & & \\
\hline & & Complicated & Noncomplicated & Complicated & Noncomplicated & & \\
\hline $\begin{array}{l}\text { End stage renal } \\
\text { disease }\end{array}$ & $\begin{array}{l}<65 \text { years; } \\
\text { female }\end{array}$ & 2 & 5 & 7 & 1370 & $75.7(6.23-570.01)$ & .01 \\
\hline $\begin{array}{l}\text { Immunosuppres- } \\
\text { sion }\end{array}$ & $\begin{array}{l}<65 \text { years; } \\
\text { female }\end{array}$ & 3 & 46 & 6 & 1329 & $14.35(2.25-69.89)$ & .03 \\
\hline $\begin{array}{l}\text { Chronic kidney } \\
\text { disease }\end{array}$ & $\begin{array}{l}<65 \text { years; } \\
\text { female }\end{array}$ & 3 & 58 & 6 & 1317 & $11.3(1.78-54.41)$ & .04 \\
\hline $\begin{array}{l}\text { Chronic kidney } \\
\text { disease }\end{array}$ & $\begin{array}{l}<65 \text { years; } \\
\text { male }\end{array}$ & 13 & 66 & 40 & 1662 & $8.16(3.82-16.5)$ & $<.001$ \\
\hline Hypertension & $\begin{array}{l}<65 \text { years; } \\
\text { male }\end{array}$ & 17 & 162 & 36 & 1566 & $4.56(2.35-8.55)$ & $<.001$ \\
\hline Obesity & $\begin{array}{l}<65 \text { years; } \\
\text { male }\end{array}$ & 25 & 359 & 28 & 1369 & $3.4(1.88-6.14)$ & .001 \\
\hline Hospitalization & $\begin{array}{l}<65 \text { years; } \\
\text { male }\end{array}$ & 21 & 285 & 32 & 1443 & $3.32(1.79-6.04)$ & .004 \\
\hline $\begin{array}{l}\text { End stage renal } \\
\text { disease }\end{array}$ & $\begin{array}{l}<65 \text { years; } \\
\text { male }\end{array}$ & 3 & 7 & 50 & 1721 & $14.67(2.38-66.53)$ & .03 \\
\hline Diabetes & $\begin{array}{l}<65 \text { years; } \\
\text { male }\end{array}$ & 9 & 105 & 44 & 1623 & $3.16(1.32-6.79)$ & .04 \\
\hline $\begin{array}{l}\text { Neurological disor- } \\
\text { ders }\end{array}$ & $\begin{array}{l}\geq 65 \text { years; } \\
\text { female }\end{array}$ & 26 & 66 & 15 & 136 & $3.55(1.68-7.74)$ & .008 \\
\hline $\begin{array}{l}\text { Chronic kidney } \\
\text { disease }\end{array}$ & $\begin{array}{l}\geq 65 \text { years; } \\
\text { female }\end{array}$ & 30 & 89 & 11 & 113 & $3.45(1.57-8.06)$ & .02 \\
\hline Home care & $\begin{array}{l}\geq 65 \text { years; } \\
\text { female }\end{array}$ & 10 & 16 & 31 & 186 & $3.72(1.38-9.69)$ & .04 \\
\hline $\begin{array}{l}\text { Other cardiovascu- } \\
\text { lar diseases }\end{array}$ & $\begin{array}{l}\geq 65 \text { years; } \\
\text { female }\end{array}$ & 15 & 33 & 26 & 169 & $2.94(1.3-6.51)$ & .04 \\
\hline $\begin{array}{l}\text { Cardiovascular } \\
\text { diseases }\end{array}$ & $\begin{array}{l}\geq 65 \text {; fe- } \\
\text { male }\end{array}$ & 19 & 48 & 22 & 154 & $2.76(1.29-5.85)$ & .045 \\
\hline $\begin{array}{l}\text { Cognitive impair- } \\
\text { ment }\end{array}$ & $\begin{array}{l}\geq 65 \text { years; } \\
\text { male }\end{array}$ & 16 & 15 & 54 & 213 & $4.18(1.81-9.72)$ & .009 \\
\hline Depression & $\begin{array}{l}\geq 65 \text { years; } \\
\text { male }\end{array}$ & 26 & 38 & 44 & 190 & $2.94(1.55-5.58)$ & .01 \\
\hline $\begin{array}{l}\text { Neurological disor- } \\
\text { ders }\end{array}$ & $\begin{array}{l}\geq 65 \text { years; } \\
\text { male }\end{array}$ & 28 & 47 & 42 & 181 & $2.56(1.38-4.73)$ & .02 \\
\hline $\begin{array}{l}\text { End stage renal } \\
\text { disease }\end{array}$ & $\begin{array}{l}\geq 65 \text { years; } \\
\text { male }\end{array}$ & 19 & 26 & 51 & 202 & $2.88(1.39-5.9)$ & .03 \\
\hline $\begin{array}{l}\text { Chronic kidney } \\
\text { disease }\end{array}$ & $\begin{array}{l}\geq 65 \text { years; } \\
\text { male }\end{array}$ & 40 & 85 & 30 & 143 & $2.24(1.26-4.02)$ & .03 \\
\hline $\begin{array}{l}\text { Fluid and elec- } \\
\text { trolyte disorders }\end{array}$ & $\begin{array}{l}\geq 65 \text { years; } \\
\text { male }\end{array}$ & 17 & 22 & 53 & 206 & $2.99(1.39-6.38)$ & .03 \\
\hline
\end{tabular}

${ }^{\mathrm{a}}$ OR: odds ratio. ORs greater than 1 suggest an increased risk for COVID-19 complications in patients with the noted condition.

${ }^{\mathrm{b}}$ In each stratum, rows are sorted ascendingly by $P$ value.

\section{Discussion}

We compared the prevalence of dozens of existing conditions in Israeli SARS-CoV-2-positive and complicated COVID-19 patient cohorts to highlight conditions associated with a high risk of complications. A few other studies have employed a similar study design to identify risk factors for COVID-19 complications. For example, Ebinger et al [8] studied a cohort of symptomatic COVID-19 individuals ( $\mathrm{N}=442)$ and examined the association of existing conditions with disease severity; and the OpenSAFELY Collaborative explored the risk of 
COVID-19-related hospital death in the general population ( $>17$ million). We emphasize that cohort composition dictates the research question it can address: our analysis focuses on SARS-CoV-2-positive individuals, hence searches for risk factors of complications in patients who already contracted the virus (but are potentially asymptomatic), while studying the general population may combine risk factors for infection and severe COVID-19 outcome. Additionally, cohorts that consider only a subset of patients, defined based on disease outcome (eg, symptomatic or hospitalized) or otherwise nonrepresentative of the entire population (eg, demographically skewed) may introduce biases to the analysis [21]; instead, we study here all SARS-CoV-2-infected patients in a large, nationwide health organization.

Multiple studies (eg, [7,22]) have shown that COVID-19 complications are most strongly associated with age and sex. Stratifying by these factors provides readily interpretable insights on the supplemental associations (in addition to older age and male sex) between pre-existing conditions and disease complications.

Many conditions highlighted by our analysis have been previously reported $[5,6,8]$ and are part of commonly used at-risk definitions [1,3], including hypertension, obesity, as well as kidney and cardiovascular diseases. We do, however, identify a few additional risk factors, notably depression in patients aged 18 - 50 years and males $\geq 65$ years; and cognitive and neurological disorders in patients $\geq 65$ years. These additions may be, in part, associated with the different age distribution in the $\geq 65$ years group (median 76 years, IQR 70-83.5 years versus 72 years, IQR 68-78 years, in the complicated and noncomplicated COVID-19 cohorts, respectively) and rely on small sample size (only 7 patients aged 18-50 years with depression in the complicated COVID-19 cohort; Table 3). Nonetheless, with some preliminary support [7], they may deserve more consideration in future studies. Our analysis also points out to the reduced importance of respiratory diseases and smoking. Both conditions appear as factors in most at-risk definitions [3,5]: chronic obstructive pulmonary disease has been associated with severe COVID-19 in multiple studies [23] (though not all [6]), while the role of smoking has been somewhat controversial $[23,24]$. The discrepancies between our analysis and previous reports likely stem from the different cohorts analyzed: SARS-CoV-2-positive individuals, ranging from asymptomatic to severe COVID-19 versus hospitalized COVID-19 patients, respectively. Other study-related attributes (eg, country-specific characteristics) may also contribute to the varying importance of the studied risk factors.
In parallel to the COVID-19 epidemiological characterization efforts, researchers have also attempted to use retrospective observational data to derive risk models for severe COVID-19 patients [25]. Such models require ample data of COVID-19 patients for both model training and performance assessment. As such data are scarce at present, some models compromised on using data for other diseases with, supposedly, similar clinical trajectory and complications. For example, DeCapprio et al [26] trained models on US Medicare claims data to predict inpatient visits with a primary diagnosis of either pneumonia, influenza, acute bronchitis, or other specified upper respiratory infections as proxy for COVID-19 complications. However, as previously reported (eg, [27]), and in agreement with our analysis, severe COVID-19 patient characteristics differ considerably from that of other diseases, thus limiting the generalizability of such models to COVID-19 and requiring adjustments to their parameters [4].

Our study has several limitations. First and foremost, it relies on routinely maintained electronic health records, which may be inaccurate and incomplete [28]. Second, the number of complicated COVID-19 patients in the MHS data is below 200, limiting the statistical power of our analysis. Third, health care policies and, in particular, testing criteria, may systematically bias the composition of the SARS-CoV-2-positive cohort. Fourth, asymptomatic and patients with mild symptoms of COVID-19 (currently in the noncomplicated cohort) may deteriorate and eventually be part of the complicated cohort, potentially modifying the results of the analysis. Fifth, our analysis is univariate in nature, testing the association of individual conditions with COVID-19 complications; as such, it is unable to uncover more complex relations (eg, interdependencies between existing conditions and COVID-19 complications), which may be discovered by multivariate analysis. Finally, we focused on data from Israel; characteristics in other geographies may differ [27]. We attempted to mitigate some of these limitations by age and sex stratification and robust estimations of statistical significance. We also note that, at the current point in time, many of these shortcomings are shared by all published research on COVID-19.

Notwithstanding these limitations, our work adopts a novel vantage point to the problem of identifying patients at increased risk for COVID-19 complications. Importantly, as SARS-CoV-2 containment efforts focus on patients at risk for severe complications (eg, shielding vulnerable population in the United Kingdom [3]), changes in the list of considered conditions may have a substantial effect on a large number of individuals, thus calling for continuous fine-tuning of the corresponding definitions.

\section{Acknowledgments}

We thank Guy Amit, Maytal Bivas-Benita, and Irena Girshovitz, KI Research Institute, and Ran Nir-Paz, Hadassah Medical Center, for insightful discussions and comments.

This research received no specific grant from any funding agency in the public, commercial, or not-for-profit sectors. 


\section{Authors' Contributions}

NK was responsible for study conceptualization; BM, KM, and YB for data curation; CY, BM, and NK for investigation and methodology; PA and VS for project administration; CY for original draft preparation; CY, BM, KM, PA, YB, and GC reviewed and edited the manuscript.

\section{Conflicts of Interest}

PA reports personal and other fees from Medial Research, unrelated to the submitted work.

\section{Multimedia Appendix 1}

Odds ratio analysis.

[XLSX File (Microsoft Excel File), 29 KB-Multimedia Appendix 1]

\section{References}

1. Centers for Disease Control and Prevention. Coronavirus Disease 2019 (COVID-19). 2020. URL: https://www.cdc.gov/ coronavirus/2019-ncov/need-extra-precautions/people-at-higher-risk.html [accessed 2020-04-21]

2. European Centre for Disease Prevention and Control. Rapid risk assessment: Coronavirus disease 2019 (COVID-19) pandemic: increased transmission in the EU/EEA and the UK - eighth update. URL: https://www.ecdc.europa.eu/en/ publications-data/rapid-risk-assessment-coronavirus-disease-2019-covid-19-pandemic-eighth-update [accessed 2020-04-21]

3. United Kingdom National Health Service. Guidance and updates for GPs: Highest clinical risk patients Internet. URL: https://www.england.nhs.uk/coronavirus/publication/guidance-and-updates-for-gps-at-risk-patients/ [accessed 2020-04-27]

4. Barda N, Riesel D, Akriv A, Levi J, Finkel U, Yona G, et al. Performing risk stratification for COVID-19 when individual level data is not available, the experience of a large healthcare organization. medRxiv 2020 Apr [FREE Full text] [doi: $10.1101 / 2020.04 .23 .20076976]$

5. Xu L, Mao Y, Chen G. Risk factors for 2019 novel coronavirus disease (COVID-19) patients progressing to critical illness: a systematic review and meta-analysis. Aging (Albany NY) 2020 Jun 23;12(12):12410-12421 [FREE Full text] [doi: 10.18632/aging.103383] [Medline: 32575078]

6. Richardson S, Hirsch JS, Narasimhan M, Crawford JM, McGinn T, Davidson KW, the Northwell COVID-19 Research Consortium, et al. Presenting Characteristics, Comorbidities, and Outcomes Among 5700 Patients Hospitalized With COVID-19 in the New York City Area. JAMA 2020 Apr 22 [FREE Full text] [doi: 10.1001/jama.2020.6775] [Medline: $\underline{32320003]}$

7. Williamson EJ, Walker AJ, Bhaskaran K, Bacon S, Bates C, Morton CE, et al. Factors associated with COVID-19-related death using OpenSAFELY. Nature 2020 Jul 08. [doi: 10.1038/s41586-020-2521-4] [Medline: 32640463]

8. Ebinger J, Achamallah N, Ji H, Claggett B, Sun N, Botting P, et al. Pre-existing traits associated with Covid-19 illness severity. PLoS One 2020;15(7):e0236240 [FREE Full text] [doi: 10.1371/journal.pone.0236240] [Medline: 32702044]

9. Shalev V, Chodick G, Goren I, Silber H, Kokia E, Heymann AD. The use of an automated patient registry to manage and monitor cardiovascular conditions and related outcomes in a large health organization. Int J Cardiol 2011 Nov 03;152(3):345-349. [doi: 10.1016/j.ijcard.2010.08.002] [Medline: 20826019]

10. Sella T, Shoshan A, Goren I, Shalev V, Blumenfeld O, Laron Z, et al. A retrospective study of the incidence of diagnosed Type 1 diabetes among children and adolescents in a large health organization in Israel, 2000-2008. Diabet Med 2011 Jan;28(1):48-53. [doi: 10.1111/j.1464-5491.2010.03174.x] [Medline: 21166845]

11. Chodick G, Heymann A, Shalev V, Kookia E. The epidemiology of diabetes in a large Israeli HMO. Eur J Epidemiol 2002 Dec;18(12):1143-1146. [doi: 10.1023/b:ejep.0000006635.36802.c8]

12. Weitzman D, Chodick G, Shalev V, Grossman C, Grossman E. Prevalence and factors associated with resistant hypertension in a large health maintenance organization in Israel. Hypertension 2014 Oct;64(3):501-507. [doi: 10.1161/HYPERTENSIONAHA.114.03718] [Medline: 24958503]

13. Goldshtein I, Nguyen AM, dePapp AE, Ish-Shalom S, Chandler JM, Chodick G, et al. Epidemiology and correlates of osteoporotic fractures among type 2 diabetic patients. Arch Osteoporos 2018 Mar 03;13(1):15. [doi: 10.1007/s11657-018-0432-x] [Medline: 29502187]

14. Yu J, Goldshtein I, Shalev V, Chodick G, Ish-Shalom S, Sharon O, et al. Renal impairment among postmenopausal women with osteoporosis from a large health plan in Israel. Arch Osteoporos 2015;10:210. [doi: 10.1007/s11657-015-0210-y] [Medline: 25753617]

15. Kodesh A, Goldshtein I, Gelkopf M, Goren I, Chodick G, Shalev V. Epidemiology and comorbidity of severe mental illnesses in the community: findings from a computerized mental health registry in a large Israeli health organization. Soc Psychiatry Psychiatr Epidemiol 2012 Dec;47(11):1775-1782. [doi: 10.1007/s00127-012-0478-9] [Medline: 22310700]

16. Metcalfe D, Masters J, Delmestri A, Judge A, Perry D, Zogg C, et al. Coding algorithms for defining Charlson and Elixhauser co-morbidities in Read-coded databases. BMC Med Res Methodol 2019 Jun 06;19(1):115 [FREE Full text] [doi: 10.1186/s12874-019-0753-5] [Medline: 31170931] 
17. Kuan V, Denaxas S, Gonzalez-Izquierdo A, Direk K, Bhatti O, Husain S, et al. A chronological map of 308 physical and mental health conditions from 4 million individuals in the English National Health Service. Lancet Digit Health 2019 Jun;1(2):e63-e77 [FREE Full text] [doi: 10.1016/S2589-7500(19)30012-3] [Medline: 31650125]

18. Agency for Healthcare Research and Quality Healthcare Cost and Utilization Project (HCUP). Clinical Classifications Software (CCS) 2015. URL: https://www.hcup-us.ahrq.gov/toolssoftware/ccs/CCSUsersGuide.pdf [accessed 2020-04-21]

19. Benjamini Y, Hochberg Y. Controlling the False Discovery Rate: A Practical and Powerful Approach to Multiple Testing. Journal of the Royal Statistical Society: Series B (Methodological) 2018 Dec 05;57(1):289-300. [doi: 10.1111/j.2517-6161.1995.tb02031.x]

20. von Elm E, Altman DG, Egger M, Pocock SJ, Gøtzsche PC, Vandenbroucke JP, STROBE Initiative. The Strengthening the Reporting of Observational Studies in Epidemiology (STROBE) statement: guidelines for reporting observational studies. J Clin Epidemiol 2008 Apr;61(4):344-349. [doi: 10.1016/j.jclinepi.2007.11.008] [Medline: 18313558]

21. Griffith G, Morris T, Tudball M, Herbert A, Mancano G, Pike L, et al. Collider bias undermines our understanding of COVID-19 disease risk and severity. medRxiv 2020 [FREE Full text] [doi: 10.1101/2020.05.04.20090506]

22. Prieto-Alhambra D, Ballo E, Coma-Redon E, Mora N, Aragon M, Prats-Uribe A, et al. Hospitalization and 30-day fatality in 121,263 COVID-19 outpatient cases. medRxiv 2020 [FREE Full text] [doi: 10.1101/2020.05.04.20090050]

23. Zhao Q, Meng M, Kumar R, Wu Y, Huang J, Lian N, et al. The impact of COPD and smoking history on the severity of COVID-19: A systemic review and meta-analysis. J Med Virol 2020 Apr 15 [FREE Full text] [doi: 10.1002/jmv.25889] [Medline: $\underline{\text { 32293753] }}$

24. Lippi G, Henry BM. Active smoking is not associated with severity of coronavirus disease 2019 (COVID-19). Eur J Intern Med 2020 May;75:107-108 [FREE Full text] [doi: 10.1016/j.ejim.2020.03.014] [Medline: 32192856]

25. Wynants L, Van Calster B, Collins GS, Riley RD, Heinze G, Schuit E, et al. Prediction models for diagnosis and prognosis of covid-19 infection: systematic review and critical appraisal. BMJ 2020 Apr 07;369:m1328 [FREE Full text] [doi: 10.1136/bmj.m1328] [Medline: 32265220]

26. DeCaprio D, Gartner J, Burgess T, Kothari S, Sayed S, McCall C. Building a COVID-19 Vulnerability Index. arXiv 2020 [FREE Full text] [doi: 10.1101/2020.03.16.20036723]

27. Burn E, You S, Sena A, Kostka K, Abedtash H, Abrahão MTF, et al. An international characterisation of patients hospitalised with COVID-19 and a comparison with those previously hospitalised with influenza. medRxiv 2020 Apr 25 [FREE Full text] [doi: 10.1101/2020.04.22.20074336] [Medline: 32511443]

28. Myers L, Stevens J. Using EHR to Conduct Outcome and Health Services Research. In: MIT Critical Data, editor. Second Anal Electron Health Rec Internet Cham: Springer International Publishing; 2016.

\section{Abbreviations}

CDC: Centers for Disease Control and Prevention

COVID-19: coronavirus disease

ECDC: European Centre for Disease Prevention and Control

MHS: Maccabi Health Services

MoH: Ministry of Health

NHS: National Health Service

OR: odds ratio

SARS-CoV-2: severe acute respiratory syndrome coronavirus 2

STROBE: Strengthening The Reporting of OBservational Studies in Epidemiology

Edited by T Sanchez; submitted 31.05.20; peer-reviewed by M Romo; comments to author 20.07.20; revised version received 26.07.20;
accepted 03.08.20; published 25.08.20
Please cite as:
Yanover C, Mizrahi B, Kalkstein N, Marcus K, Akiva P, Barer Y, Shalev V, Chodick G
What Factors Increase the Risk of Complications in SARS-CoV-2-Infected Patients? A Cohort Study in a Nationwide Israeli Health
Organization
JMIR Public Health Surveill 2020;6(3):e20872
URL: $\underline{\text { http://publichealth.jmir.org/2020/3/e20872/ }}$
doi: $10.2196 / 20872$
PMID: $\underline{32750009}$

(C) Chen Yanover, Barak Mizrahi, Nir Kalkstein, Karni Marcus, Pinchas Akiva, Yael Barer, Varda Shalev, Gabriel Chodick. Originally published in JMIR Public Health and Surveillance (http://publichealth.jmir.org), 25.08.2020. This is an open-access 
article distributed under the terms of the Creative Commons Attribution License (https://creativecommons.org/licenses/by/4.0/), which permits unrestricted use, distribution, and reproduction in any medium, provided the original work, first published in JMIR Public Health and Surveillance, is properly cited. The complete bibliographic information, a link to the original publication on http://publichealth.jmir.org, as well as this copyright and license information must be included. 